\section{COMITÊS HOSPITALARES DE PREVENÇÃO DE MORTE MATERNA EM FORTALEZA: PERFIL E FUNCIONAMENTO}

\author{
Hospital Committees for the Prevention of Maternal Mortality \\ in Fortaleza: Profile and Functioning \\ Comités hospitalarios para la prevención de la muerte materna \\ de Fortaleza: el perfily su funcionamiento
}

\begin{abstract}
RESUMO
Objetivo: Caracterizar os Comitês Hospitalares de Prevenção de Morte Materna, na cidade de Fortaleza-CE, quanto ao perfil de seus profissionais e à metodologia de funcionamento. Métodos: Estudo descritivo, de corte transversal, com abordagem quantitativa, realizado no período de março a setembro de 2013, pesquisando-se todos os Comitês Hospitalares existentes em Fortaleza-CE, por meio de entrevistas com os profissionais responsáveis, no total de oito. Utilizou-se um questionário estruturado contendo 46 perguntas, com todas as variáveis baseadas no Manual dos Comitês de Morte Materna - 2007, elaborado pelo Ministério da Saúde. Resultados: Os comitês são formados por médicos, enfermeiras, fisioterapeutas e assistente social. Nenhum possui estrutura física própria para seu funcionamento e os profissionais não têm dedicação exclusiva nem recebem remuneração para exercer as atividades. Dois comitês possuem regimento, dois se reúnem mensalmente, um quinzenalmente e um não tem data fixa para se reunir. Todos realizam a investigação hospitalar dos óbitos maternos e dos óbitos de mulher em idade fértil, discutem os casos com outros profissionais e realizam a divulgação das informações por meio de relatórios. Conclusão: Os Comitês Hospitalares de Prevenção de Morte Materna em Fortaleza estão organizados conforme a realidade de cada hospital, compostos com caráter multiprofissional, com faixa etária predominante de 41 a 59 anos, cujo processo de trabalho está voltado para a vigilância epidemiologia dos óbitos materno, fetal e infantil, bem como da evitabilidade destes, o que os caracteriza como comitês funcionantes e atuantes.
\end{abstract}

Descritores: Comitê de Profissionais; Morte Materna; Vigilância Epidemiológica.

\section{ABSTRACT}

Objective: To characterize the Hospital Committees for Maternal Mortality Prevention in the city of Fortaleza, CE, in relation to the profile of its professionals and the functioning methodology. Methods: A quantitative descriptive cross-sectional study conducted from March to September 2013 to research all the existing Hospital Committees in Fortaleza, CE, through interviews with the professionals in charge, resulting in a total of eight. We used a structured questionnaire containing 46 questions, with all variables being based on the 2007 Maternal Mortality Committees Manual designed by the Ministry of Health. Results: The committees are made up of physicians, nurses, physiotherapists and social workers. None has its own physical structure for its functioning, and all professionals do not have exclusive dedication or receive any compensation for performing the activities. Two committees are regulated, two hold meetings every month, one every two weeks, and one has not a set up date to meet. All of them carry out hospital investigation of maternal deaths and deaths of women of childbearing age, discuss cases with other professionals, and perform the dissemination of information through reports. Conclusions: The Hospital Committees for the Prevention of Maternal Mortality in Fortaleza are organized according to the reality of each hospital on a multidisciplinary basis and are predominantly composed of individuals aged 42-59 years, whose working process focuses on the epidemiological surveillance of maternal, fetal and infant deaths and their prevention, which characterizes them as active and functioning committees.

Descriptors: Professional Staff Committees; Maternal Death; Epidemiological Surveillance.
Artigo Original
1) Faculdade Metropolitana da Grande Fortaleza - FAMETRO - Fortaleza (CE) Brasil

2) Universidade Federal do Ceará - UFC Fortaleza (CE) - Brasil

3) Universidade de Fortaleza - UNIFOR Fortaleza (CE) - Brasil
Recebido em: 06/05/2015 Revisado em: 18/05/2015 Aceito em: 30/05/2015 


\section{RESUMEN}

Objetivo: Caracterizar los Comités Hospitalarios para la prevención de la muerte materna en la ciudad de Fortaleza$C E$ respecto el perfil de sus profesionales y la metodología de funcionamiento. Métodos: Estudio descriptivo, de corte transversal y abordaje cuantitativo realizado entre el periodo de marzo y septiembre de 2013, investigándose todos los Comités Hospitalarios de Fortaleza-CE a través de entrevistas con los profesionales responsables, en un total de ocho. Se utilizó un cuestionario estructurado con 46 preguntas y las variables basadas en la Guía de los Comités de la Muerte Materna 2007, elaborado por el Ministerio de la Salud. Resultados: Los comités se componen de médicos, enfermeras, fisioterapeutas y trabajador social. Ningún tiene estructura fisica propia para su funcionamiento y los profesionales no tienen dedicación exclusiva ni reciben remuneración para ejercer sus actividades. Dos de ellos tienen su reglamento, dos se reúnen cada mes, uno cada quince días y uno no tiene fecha determinada para reunirse. Todos realizan la investigación hospitalaria de las muertes maternas y las de la mujer con edad fértil, discuten los casos con otros profesionales y realizan la divulgación de las informaciones a través de informes. Conclusión: Los Comités Hospitalarios para la prevención de la muerte materna de Fortaleza están organizados según la realidad de cada hospital, de carácter multiprofesional y franja de edad entre 41 y 59 años cuyo el proceso de trabajo está direccionado para la vigilancia epidemiológica de las muertes maternas, fetal e infantil así como de su evitabilidad, lo que les caracterizan como comités que funcionan y son activos.

Descriptores: Comités de Profesionales; Muerte Materna; Vigilancia Epidemiológica.

\section{INTRODUÇÃO}

As primeiras experiências sobre Comitês de Prevenção de Morte Materna datam de 1931, quando foram criados os primeiros comitês na Filadélfia, e um ano depois em Nova Iorque, ambos nos Estados Unidos da América ${ }^{(1)}$. Embora estes tenham sido os primeiros comitês do mundo a trabalharem com a investigação e a prevenção das mortes maternas, a experiência dos comitês do Reino Unido e de Cuba é bastante referida por meio de seus resultados positivos no desenvolvimento de medidas eficazes para a prevenção dos óbitos maternos ${ }^{(2)}$.

No Brasil, a implantação dos comitês foi uma estratégia adotada pelo Ministério da Saúde após implantado o PAISM (Programa de Assistência Integral à Saúde da Mulher), em $1983^{(1)}$, tendo os pioneiros e mais bem-sucedidos comitês implementados no Paraná e em São Paulo, no começo dos anos $1990^{(3-5)}$, ambos considerados atuantes até os dias atuais.
Segundo o Ministério da Saúde, os comitês são organismos de natureza interinstitucional, multiprofissional e confidencial que visam analisar todos os óbitos maternos e apontar medidas de intervenção para a sua redução na região de abrangência ${ }^{(1)}$.

Os municípios e instituições de saúde têm autonomia para organizar seus comitês, mas o que comumente acontece no Brasil é a criação dos comitês municipais e hospitalares com base nos Comitês Estaduais de Morte Materna ${ }^{(6)}$.

A morte materna é um desafio para a saúde pública, uma vez que são vidas que poderiam ter sido salvas se tivessem sido tomadas as devidas providências em tempo hábil e oportuno ${ }^{(7)}$.

Estudar os Comitês de Óbitos Maternos faz-se essencial para a construção de estratégias para o enfrentamento desse problema, uma vez que a mortalidade materna reflete a situação social na qual vivem as mulheres de uma sociedade. Além disso, os comitês, quando funcionam adequadamente e são atuantes, poderiam colaborar para a identificação de falhas durante assistência prestada à gestante, puérpera e recém-nascido, o que é de fundamental importância para a correção de possíveis inadequações e redução da morbimortalidade materna e infantil ${ }^{(8,9)}$.

Há poucos dados sobre a atuação dos Comitês de Prevenção da Morte Materna no Brasil, e nenhum sobre os comitês do estado do Ceará. Assim, o presente estudo tem como objetivo caracterizar os Comitês Hospitalares de Prevenção de Morte Materna, na cidade de Fortaleza-CE, quanto ao perfil de seus profissionais e a metodologia de funcionamento.

\section{MÉTODOS}

Estudo descritivo, de corte transversal, com abordagem quantitativa, realizado no município de Fortaleza-CE, no período de março a setembro de 2013. Participaram do estudo todos os Comitês Hospitalares de Prevenção de Morte Materna (CHPMM) existentes, no total de quatro. Dentre estes, estão os comitês dos três principais hospitais/ maternidades de referência para gestação de alto risco da capital cearense. Três são hospitais terciários, dois pertencentes são à rede pública de saúde do estado do Ceará e um hospital-escola é vinculado à Universidade Federal do Ceará. O quarto hospital é classificado como secundário e pertence à rede pública do município de Fortaleza.

Entrevistaram-se oito profissionais atuantes nos referidos comitês. Para a primeira aproximação com os participantes da pesquisa, realizou-se contato via telefone com o(a) coordenador(a) de cada comitê, a fim de explicar a pesquisa e obter autorização para contatar, em seguida, 
os demais membros. Todas as ligações foram feitas pela mesma pesquisadora, utilizando-se um único roteiro de abordagem.

Naquela ocasião, cada CHPMM foi convidado a participar da pesquisa e realizaram-se as devidas apresentações sobre o estudo, explicando os objetivos, relevância e justificativa, sendo dada a oportunidade aos coordenadores para esclarecerem quaisquer dúvidas sobre a investigação. Após confirmação de interesse em participar da pesquisa, agendou-se dia, local e horário para encontro, de acordo com a disponibilidade dos participantes. Para realizar cada entrevista, houve um agendamento prévio. No momento do segundo contato, feito pessoalmente com cada participante da pesquisa, houve a leitura e assinatura do Termo de Consentimento Livre e Esclarecido.

Utilizou-se um questionário estruturado, contendo 46 perguntas, dividido em três partes. A primeira parte referese a dados relacionados aos entrevistados, com o objetivo de traçar o perfil dos membros dos comitês. A segunda parte é referente aos dados de identificação e caracterização do comitê: procedência, classificação, data da constituição, data do início das atividades, quantidade de profissionais, quantidade de profissionais exclusivos, instituição ou órgão que os profissionais representam e estrutura física. $\mathrm{O}$ terceiro bloco de perguntas refere-se a informações sobre o funcionamento do comitê: informações sobre a periodicidade das reuniões, regimento, investigação e análise dos óbitos, recursos utilizados para as investigações, elaboração de relatórios, capacitações, financiamento e divulgação dos dados. As variáveis utilizadas foram baseadas no Manual dos Comitês de Mortalidade Materna (MCMM), elaborado pelo Ministério da Saúde em sua terceira e última edição, no ano de 2007, que normatiza todas as questões referentes à composição, funcionamento e objetivos dos CPMM.

Para complementação dos dados, utilizaram-se documentos concedidos pelos próprios comitês, como atas das reuniões, relatórios e boletins informativos.

Os dados foram organizados no programa Excel, para melhor visualização das informações coletadas.

Este trabalho obedeceu às exigências éticas e cientificas de acordo com as Diretrizes e Normas Regulamentadoras de Pesquisa Envolvendo Seres Humanos do Conselho Nacional de Saúde, Resolução 466/2012. Foram submetidos aos Comitês de Ética em Pesquisa: da MEAC (Parecer $n^{\circ}$ 197.294, favorável, em 15/02/2013); do HGF (Parecer favorável, em 10/04/2013); e do HGCC (Parecer n 262.877, favorável, em 03/05/2013). Além disso, a pesquisa recebeu autorização do Hospital Gonzaguinha de Messejana, por meio de carta de anuência assinada por seus gestores. Todos os participantes assinaram o Termo de Consentimento Livre e Esclarecido (TCLE), elaborado conforme exigência da Resolução 466/2012 do Conselho Nacional de Saúde.

\section{RESULTADOS}

Do total de quatro comitês avaliados, três são exclusivamente maternos e um materno-infantil e fetal. Constituíram-se entre 1999 e 2006 e iniciaram suas atividades entre 1999 e 2005, com tempo médio de atuação de 10,5 anos (variando de 8 a 14 anos).

$\mathrm{O}$ total de profissionais que compõem os comitês variou de 4 a 16, com média de 8,5 membros. Do total, $14(85,7 \%)$ profissionais atuam nos comitês de 0 a 5 anos e dois (14,3\%), de 6 a 10. A enfermagem (enfermeiros) apresentou-se como categoria profissional predominante $(57,1 \% ; n=9)$, do sexo feminino $(84,7 \% ; n=13)$, com faixa etária de 41 a 59 anos $(71,4 \% ; n=11)$. Nenhum profissional dedica exclusivamente suas atividades aos comitês. Com relação à composição profissional, todos são formados por pelo menos médico(a) s e enfermeiro(a)s. As outras categorias profissionais que participam são técnico de enfermagem, assistente social e fisioterapeuta.

Os membros são representantes da vigilância epidemiológica e da área da obstetrícia de cada hospital, sendo que um comitê, por ser materno-infantil e fetal, é formado também por profissionais da área da pediatria e neonatologia. Apenas um comitê referiu a participação de profissionais da universidade, provavelmente por se tratar de um hospital-escola.

Nenhum comitê possui estrutura física exclusiva para realização das atividades. Dessa forma, em três comitês, os profissionais se reúnem na sala da vigilância epidemiológica, e o outro utiliza o centro de estudos do hospital para desenvolver suas atividades.

No que diz respeito ao regimento interno, dois o possuem. A periodicidade das reuniões é quinzenal em dois comitês, e mensal em um. O outro não possui data fixa para se reunir.

Todos os comitês realizam a investigação de óbito materno e de mulheres em idade fértil, e sempre utilizam para isso informações contidas na declaração de óbito e nos prontuários hospitalares. Os prontuários ambulatoriais são utilizados apenas por um comitê, dois recorrem aos profissionais que atenderam a paciente que foi a óbito frequentemente e um sempre. As informações dos familiares são frequentemente usadas por um comitê e raramente por dois. Os sistemas de informação mais usados pelos comitês são o Sistema de Informações sobre Mortalidade (SIM) e o Sistema Nacional de Nascidos Vivos (SINASC).

Todos os comitês realizam a classificação quanto à evitabilidade dos óbitos. No entanto, quando indagados sobre a quantidade de óbitos evitáveis, não evitáveis e não classificados, no ano de início das atividades e no último ano, um comitê não soube informar. 
Apenas um comitê respondeu ao total de óbitos maternos no início das atividades; para o último ano (2012), todos responderam. Ocorreram de 1 a 17 óbitos maternos no ano de 2012 nos comitês investigados, com média de 10 óbitos maternos por hospital.

Todos os comitês elaboram relatórios de suas atividades e divulgam seus dados e informações, em periodicidade que varia de mensal a anual.

Quando perguntados sobre a discussão dos casos com os membros dos comitês e com profissionais que não os compõem, todos responderam realizar. Três comitês realizam capacitação com os profissionais do hospital sobre a problemática da mortalidade materna. Três comitês elaboram material didático com propostas de intervenção para a redução da morte materna. Todos os profissionais que responderam a presente pesquisa referiram realizar a mobilização de parceiros com a finalidade de garantir a execução das medidas propostas.

Os membros dos comitês hospitalares investigados participam das reuniões de outros comitês nos planos municipal e/ou estadual.

\section{DISCUSSÃO}

A organização dos comitês hospitalares é considerada imprescindível no sentido de representar um espaço para discussão entre os profissionais de saúde e gestores do hospital, viabilizando, por meio da reflexão e estudo dos óbitos ocorridos no estabelecimento de saúde, a identificação das responsabilidades e de medidas para evitar novos óbitos ${ }^{(5)}$. É requisito para funcionamento de maternidades que fazem parte do Programa de Gestação de Alto Risco e de Humanização do Pré-Natal e Nascimento do Ministério da Saúde e da Iniciativa Hospital Amigo da Criança ${ }^{(6,7)}$. Em Fortaleza, as três principais maternidades referência para a gestação de alto risco possuem comitês hospitalares de morte materna funcionantes.

A faixa etária da maioria dos participantes do presente estudo foi de 41 a 59 anos, evidenciando que os comitês são formados por profissionais com mais tempo de experiência. Esse achado corrobora com um estudo realizado em um Comitê Municipal de Morte Materna e Infantil em Minas Gerais, em que metade dos participantes tinha acima de dez anos de formação ${ }^{(8)}$.

Observou-se na atual pesquisa que os comitês são constituídos predominantemente por enfermeiras, no entanto, é importante destacar a participação de outros profissionais: médico(a)s, fisioterapeutas, assistentes sociais e técnicos de enfermagem. A participação de outras categorias profissionais agrega valor às discussões de cada óbito, pois permite visualizar diferentes situações de vulnerabilidade individuais ou coletivas, sociais, contextuais e programáticas nas quais as pacientes que foram a óbito estavam susceptíveis ${ }^{(9)}$. Além disso, a participação dos profissionais médicos é imprescindível para a análise das causas dos óbitos e da sua evitabilidade( ${ }^{(2)}$.

É importante destacar que a escolha dos membros dos comitês deve atender à realidade de cada hospital, entretanto, devem participar, em geral, representantes da direção clínica e profissionais das equipes de obstetrícia, pediatria e neonatologia, enfermagem, comissão de controle de infecção hospitalar $(\mathrm{CCIH})$ e núcleo de vigilância epidemiológica $^{(1,7)}$. Na presente pesquisa, os achados evidenciaram que apenas a $\mathrm{CCIH}$ não participava de nenhum comitê hospitalar de prevenção de morte materna.

As datas de constituição dos comitês são relativamente recentes no presente estudo. Elas se assemelham com o período de criação de outros comitês no país e no próprio estado do Ceará( ${ }^{(4,10)}$. Os primeiros comitês implantados oficialmente na capital do estado datam de $1999^{(4)}$, confirmando o encontrado na presente pesquisa.

O total de profissionais por comitê de prevenção de morte materna variou de 4 a 16 no atual estudo. Essa diferença se justifica pelo fato de que um dos comitês participantes do estudo possui, além do componente materno, os componentes infantil e fetal, sendo composto, também, por profissionais das áreas de pediatria e neonatologia. A equipe mínima para formação de um Comitê Hospitalar de Prevenção da Mortalidade Materna deve ser composta por um enfermeiro com conhecimento em ginecologia e obstetrícia, e um representante técnico da área de Epidemiologia ${ }^{(8)}$.

Os membros dos comitês do presente estudo reúnemse periodicamente, mas um comitê referiu não ter data fixa, reunindo seus membros conforme a demanda para investigação e análise dos óbitos. As reuniões sistemáticas dos membros dos comitês são importantes para que suas equipes analíticas estudem todos os óbitos maternos ocorridos em âmbito hospitalar. No entanto, o problema da falta de recursos humanos exclusivos, evidenciado também em outro estudo, realizado em um Comitê de Prevenção do Óbito Infantil em São Paulo(11), caracteriza a real situação de funcionamento dos comitês hospitalares em Fortaleza e, provavelmente, em todo o país. O que ocorre é a dedicação voluntária dos participantes ${ }^{(8)}$, ou seja, muitas vezes os profissionais que compõem os comitês são aqueles mais sensibilizados com a problemática da mortalidade materna ou que ensejam dedicar parte de seu tempo ao desempenho de tais atividades, como encontrado na presente pesquisa.

Em um estudo realizado em Minas Gerais, percebeuse que o trabalho no CHPMM é desempenhado por alguns membros como uma atividade voluntária, portanto, não se 
tem o estímulo financeiro para desenvolver as atividades próprias do comitê, o que dificulta a adesão de outras categorias profissionais ${ }^{(8)}$. Além disso, devido a essa atividade voluntária, os membros do comitê possuem outros vínculos empregatícios, o que diminui a carga horária disponível para participação no comitêt( ${ }^{(8)}$.

Evidenciou-se, no atual estudo, que os comitês apresentam carência de recursos infraestruturais devido ao fato de nenhum deles possuir estrutura física própria, com recursos materiais mínimos para desenvolver suas atividades. Esse fato, caracterizado pela precariedade de recursos infraestruturais, é considerado um dos obstáculos responsáveis pela fraca inserção institucional dos comitês ${ }^{(12)}$. O empenho e a dedicação dos membros dos comitês são facilitadores e garantem a manutenção, o funcionamento e a continuidade dos comitês ${ }^{(13)}$.

Outro ponto importante a ser destacado é a formalização dos comitês por meio de documentos institucionais oficiais. No presente estudo, encontrou-se que metade dos comitês hospitalares não possui regimento. Regulamentar legalmente a estrutura dos comitês é importante por constituir a eles um status jurídico-legal e administrativo mais consistente ${ }^{(3,8)}$, além de definir de forma clara e objetiva as atribuições de cada membro.

No que diz respeito à investigação epidemiológica dos óbitos em mulher em idade fértil e óbitos maternos, todos os realizam no atual estudo. A investigação epidemiológica é realizada a nível hospitalar, e para realizá-la, os profissionais utilizam como base as informações contidas nas declarações de óbito ${ }^{(9)}$. Essa fonte de informação contêm informações fundamentais para a investigação dos $\operatorname{casos}^{(9)}$. No entanto, a entrevista com a família durante a visita domiciliar e com os profissionais de saúde que atenderam às pacientes gestantes, em parto e/ou puerpério, também pode colaborar com a completude de dados durante a investigação ${ }^{(9)}$.

Em geral, as investigações são realizadas juntamente com os profissionais que atuam na vigilância epidemiológica de cada hospital e são relevantes para a compreensão das circunstâncias em que ocorreram os óbitos ${ }^{(7)}$. É importante ressaltar que a investigação realizada pelos comitês hospitalares é feita somente no ambiente hospitalar, nos comitês analisados do atual estudo. Sendo assim, os indicadores ambulatoriais e familiares, dados importantes que irão completar a investigação de cada caso, são encaminhados para os comitês municipais completarem ${ }^{(9)}$.

Assumir ou apontar responsáveis a nível pessoal, profissional ou institucional para os óbitos maternos faz parte das atribuições dos comitês $^{(14)}$. A classificação da evitabilidade dos óbitos é sistematicamente realizada pelos comitês. No entanto, apenas um dos comitês participantes da pesquisa em questão soube responder o total de óbitos classificados em evitáveis e não evitáveis no início das atividades do comitê. O objetivo da análise da evitabilidade dos óbitos maternos é sistematizar as informações obtidas de múltiplas fontes e ao mesmo tempo avaliar a efetividade dos serviços ${ }^{(7)}$.

Investigar e analisar as causas de mortes maternas, principalmente aquelas que poderiam ter sido evitadas, é uma atribuição que compete aos comitês municipais e hospitalares ${ }^{(15)}$, e geralmente essas atividades são realizadas em parceria com a equipe de vigilância epidemiológica do hospital e dos municípios, e com as equipes de saúde da família dos municípios ${ }^{(9)}$. Conceber os comitês como uma estratégia de vigilância epidemiológica significa assinalar a dimensão técnica de sua atuação no contexto dos processos de trabalho pertinentes ao sistema de informação em saúde ${ }^{(3)}$.

Os hospitais de referência para a gestação de alto risco apresentaram, no ano de 2012, um maior número de mortes maternas, provavelmente pelo fato de serem referência, municipal e estadual no atendimento às gestantes de alto risco, que muitas vezes chegam às instalações já em estados críticos de saúde. Um estudo realizado no Paraná sobre as causas de morte materna segundo o nível de complexidade hospitalar evidenciou que a assistência hospitalar adequada poderia ter contribuído para a evitabilidade de uma proporção significativa de óbitos maternos no Paraná durante os períodos estudados ${ }^{(16)}$.

A divulgação dos resultados, realizada pelos comitês, deve alcançar principalmente as autoridades de saúde $\mathrm{s}^{(3,6,9)} \mathrm{e}$, no caso dos hospitais, os gestores e as entidades científicas, escolas médicas, de enfermagem e demais profissionais de saúde, bem como a sociedade de uma forma geral $^{(7)}$.

No período de 1998 a 2013, foram confirmadas 1.892 mortes maternas por causas obstétricas diretas, indiretas, não obstétricas, não especificadas e tardias, sendo 1.724 por causas obstétricas diretas ou indiretas, com uma média da razão da mortalidade materna (RMM), no período, de 78,9 mortes maternas por 100.000 nascidos vivos, índice considerado alto segundo parâmetros da $\mathrm{OMS}^{(17)}$.

Um estudo realizado no Ceará em 2012 identificou que a magnitude da mortalidade materna se relaciona com as deficiências no acesso e na qualidade dos serviços e ações de saúde, e com a precariedade das medidas de prevenção e promoção da saúde reprodutiva e sexual ${ }^{(13)}$. Dessa forma, os comitês devem atentar também para aquelas mulheres em condições de near miss materno, que são aquelas mulheres em quadro de complicações graves em obstetrícia ${ }^{(15)}$, e trabalhar na perspectiva de identificar as causas das morbidades, evitando, sobretudo, o óbito. Essas mulheres, às quais se pode atribuir o conceito near miss, são de grande interesse para o estudo da morbimortalidade materna, uma vez que constituem um grupo muito mais frequente e com melhor fonte de informação - pois estão vivas - do que os casos de morte materna por $\mathrm{si}^{(15)}$. 
É preciso, no entanto, ter a compreensão de que a importância dos comitês vai muito além da função de vigilância epidemiológica. Identificar e recomendar estratégias e medidas de atenção à saúde necessárias para a redução da mortalidade materna ${ }^{(7)}$ é uma das atribuições dos comitês, bem como mobilizar parceiros e articular com os gestores responsáveis, objetivando garantir a execução das medidas apontadas.

As recomendações devem ser elaboradas de forma institucionalizada e continuada ${ }^{(4)}$, daí a importância da participação de vários agentes e perspectivas, com relevância no enfoque do trabalho interdisciplinar. É consenso entre os autores que um dos elementos-chave para as medidas de prevenção de óbitos maternos é a revisão dos casos pelos Comitês de Morte Materna, os quais devem adotar uma postura atuante e participativa ${ }^{(2,3,8,9-11,18)}$. Todos que tiveram participação direta ou indireta no óbito da mulher devem ser questionados para identificação dos fatores implicados na morte, e os casos devem ser estudados por profissionais qualificados que tenham o compromisso de educar, e não punir ${ }^{(17)}$

Articular saberes objetivando a qualificação da assistência é fundamental para ir ao encontro das necessidades e anseios da comunidade ${ }^{(19)}$; dessa forma, os comitês têm papel fundamental na divulgação de informações e educação em saúde.

\section{CONCLUSÃO}

Os Comitês Hospitalares de Prevenção de Morte Materna em Fortaleza estão organizados conforme a realidade de cada hospital, compostos com caráter multiprofissional, dos quais participam médicos, enfermeiros, fisioterapeuta e assistente social, com faixa etária predominante de 41 a 59 anos. Os profissionais são representantes da direção clínica e das equipes de obstetrícia, pediatria e neonatologia, enfermagem e do núcleo de vigilância epidemiológica.

O processo de trabalho comum a todos os comitês está voltado para a vigilância epidemiológica do óbito materno e dos óbitos fetal e infantil, bem como para a classificação da evitabilidade dos óbitos investigados. Para isso, os profissionais cumprem uma agenda regular de reuniões.

Foi evidenciada a falta de profissionais e de estrutura física para a realização das atividades dos comitês, no entanto, observou-se que os comitês mantêm suas atividades de forma regular, o que os caracteriza como funcionantes e atuantes.

\section{REFERENCIAS}

1. Ministério da Saúde (BR), Secretaria de Atenção à Saúde, Departamento de Ações programáticas estratégicas: manual dos comitês de mortalidade materna. Brasília: Ministério da Saúde; 2007.

2. Soares VMN, Martins AL. A trajetória e experiência dos Comitês de Prevenção da Mortalidade Materna do Paraná. Rev Bras Saúde Matern Infant. 2006;6(4): 453-60.

3. Rodrigues Ana Verônica; Siqueira Arnaldo A Franco de. Uma análise da implementação dos comitês de estudos de morte materna no Brasil: um estudo de caso do Comitê do Estado de São Paulo. Cad Saúde Pública. 2003;19(1):183-9.

4. Alencar Júnior CA. Os elevados índices de mortalidade materna no Brasil: razões para sua permanência. Rev Bras Ginecol Obstet. 2011;28(7):1-3.

5. Viana RC, Novaes MRCG, Calderon IMP. Mortalidade materna: uma abordagem atualizada. Com Ciências Saúde. 2011;22(Supl 1):S141-52.

6. Bittencourt SDA, Dias MAB, Wakimoto MD. Vigilância do óbito materno, infantil e fetal e atuação em comitês de mortalidade. Rio de Janeiro: Fiocruz; 2013.

7. Ministério da Saúde (BR), Secretaria de Vigilância em Saúde, Departamento de Análise de Situação de Saúde. Guia de vigilância epidemiológica do óbito materno. Brasília: Ministério da Saúde; 2009.

8. Santos CA, Silva T, Lima EC. Trajetória de um comitê de mortalidade materna. Rev Enfermagem Integrada. 2009;2(2):333-44.

9. Araújo LB. Análise da implementação e operacionalização dos comitês de prevenção do óbito infantil e fetal no estado do Ceará [dissertação]. Fortaleza: Universidade Estadual do Ceará: Universidade Estadual do Ceará; 2012.

10. Venâncio SI, Paiva R. O processo de implantação dos comitês de investigação do óbito infantil no Estado de São Paulo. Rev Bras Saúde Matern Infant. 2010;10(3):369-75.

11. Orlandi MHF. Comitês de prevenção da mortalidade materna e infantil no Paraná: estratégia de sucesso na vigilância da saúde de mulheres e crianças. Ciênc Cuid Saúde. 2008;7(1):7-8.

12. Herculano MMS, Veloso LS, Teles LMR, Oriá MOB, Almeida PC, Damasceno AKC. Óbitos maternos em uma maternidade pública de Fortaleza: um estudo epidemiológico. Rev Esc Enferm USP. 2012;46(2): 295-301.

13. Morse ML, Fonseca SC, Barbosa MD, Calil MB, Eyer FPC. Mortalidade materna no Brasil: o que mostra a 
produção científica nos últimos 30 anos? Cad Saúde Pública. 2011;27(4):623-38.

14. Morse ML, Fonseca SC, Gottgtroy CL, Waldmann CS, Gueller E. Morbidade Materna Grave e Near Misses em Hospital de Referência Regional. Rev Bras Epidemiol. 2011;14(2):310-22.

15. Soares VMN, Souza KV, Azevedo EMM, Possebon CR, Marques FF. Causas de mortalidade materna segundo nível de complexidade hospitalar. Rev Bras Ginecol Obstet. 2012;34(12):536-43.

16. Secretaria da Saúde do Estado do Ceará (BR). Mortalidade Materna. Fortaleza: Secretaria da Saúde; 2014. (Informe Epidemiológico).

17. World Health Organization - WHO, United Nations Children's Fund - UNICEF. United Nations Fund for Population Activities - UNFPA, World Bank Trends in maternal mortality: 1990 to 2008 [Internet]. Geneva: WHO; 2010 [acesso em 2015
Out 1]. Disponível em: http://whqlibdoc.who.int/ publications/2010/9789241500265_eng.pdf

18. Souza JP. Mortalidade materna no Brasil: a necessidade de fortalecer os sistemas de saúde. Rev Bras Ginecol Obstet. 2011;33(10):273-9.

19. Souza MAS, Lopes NAR, Borges FV. A importância do enfermeiro da estratégia saúde da família para a redução da morbimortalidade materna. Rev Cient Interdisciplinar. 2014;1(1):79-88.

\section{Endereço para correspondência:}

Helânia do Prado Cruz

Faculdade Metropolitana da Grande Fortaleza

Rua Conselheiro Estelita, 264

Bairro: Centro

CEP: 60.010-260 - Fortaleza - CE - Brasil

E-mail: helaniadoprado@hotmail.com 